\title{
Retrospective view on the last half century
}

The year 2014 is a significant anniversary. Personally, it is 80 years since I was born. More importantly, it is one hundred years since the outbreak of the World War I, a catastrophe from which most of the world's present problems resulted. The slaughter was bad enough but without this bloodbath we might have been spared Lenin's paradise, the great depression, Germany's embrace of an evil maniac, which occurred roughly at the time of the above mentioned birthday, the continuing spectre of a nuclear holocaust, and forty years of the cruel and smothering rule of the heart of Europe by a totalitarian regime. However, although often distressing, humanity's random walk has been forward. For example, an important paper by Ornstein and Zernike in the theory of liquids appeared in 1914 . We can only hope that the positive direction of our random walk continues.

Of course, the development of condensed matter physics occurred within these global events and is a subset of them. I have already mentioned Ornstein and Zernike. Eighty years ago (or even fifty years ago) condensed matter physics really meant solid state physics or even more narrowly electrons in metals and semi-conductors. The physics of solids (and gases) was well understood but there was no adequate theory of the liquid state. In retrospect, this is not true. The van der Waals theory is the basis of satisfactory theory but this was not understood at the time. Unfortunately, nearly a century was wasted by attempting to adjust the van der Waals parameter $a$ instead of refining the concept of an excluded volume. In fact, as it is apparent from $\mathrm{CO}_{2}$, it is not even physically necessary for a liquid phase to exist. Of course, life as we know it would be impossible without liquids, in particular water.

The beginning of our understanding of the liquid state occurred fifty years ago. Computers and simulations were being developed. This made it possible to separate the questions of the appropriateness of a model for the intermolecular potentials and the accuracy of the theoretical approximations. LonguetHiggins and Widom published an important paper that indicated that the van der Waals theory was not merely an interesting pedagogical tool but was the basis of a useful theory. In 1964, I was preparing to spend a year with John Barker in Australia. During this stay, which occurred two years later, a quantitatively accurate theory, called perturbation theory, was developed. The two important papers written in Australia and a seminal article in Reviews of Modern Physics (1976) began the development of our understanding. This should be supplemented by review articles in Science by Widom (1967) and by Chandler, Weeks, and Andersen (1983).

In my review with Barker, we asked the question, "What is liquid?”. This was a paraphrase of questions that were often asked by aliens in the television series, "Star Trek". Using the occasion of all above mentioned anniversaries, together with my colleagues from Prague and Lviv, we feel it is timely for a collection of expert contributions to access could we now answer the question "What is liquid?" firmly in the affirmative? Do we understand how surfaces affect liquids that are confined? How studies of bulk and confined liquids were influenced by the papers of van der Waals, Ornstein and Zernike, and other giants? More precisely, with this essay and on behalf of the co-editors, namely, Myroslav Holovko, Ivo Nezbeda, and Andrij Trokhymchuk, I wish to announce the special issue of Condensed Matter Physics to discuss how we arrived here, what is our present state of knowledge, and, most difficultly, where we might be heading. The issue will be published by the end of this year-early the next year, depending how efficient we will be, while deadline for submission of the selected contributions is before the end of September. The selection will be made based on the submitted titles and a brief description of the proposed contributions that must reach the Editors of the issue (cmp-liquid@icmp.lviv.ua) before April 20. There is no 
formal page limit but authors are encouraged to point out the desired page size in the description of their contribution. An invitation to submit a paper with a page limit recommendation will be sent by the end of April.

Doug Henderson

Editor of Condensed Matter Physics,

Provo UT, USA 